\title{
Graph Search with Appearance and Shape Information for 3-D Prostate and Bladder Segmentation ${ }^{\star}$
}

\author{
Qi Song ${ }^{1}$, Yinxiao Liu ${ }^{1}$, Yunlong Liu ${ }^{1}$, Punam K. Saha ${ }^{1}$, \\ Milan Sonka ${ }^{1,2,3}$, and Xiaodong $\mathrm{Wu}^{1,2}$ \\ ${ }^{1}$ Department of Electrical \& Computer Engineering, \\ ${ }^{2}$ Department of Radiation Oncology, \\ ${ }^{3}$ Department of Ophthalmology \& Visual Sciences, University of Iowa, \\ Iowa City, IA 52242, USA \\ \{qi-song, xiaodong-wu, milan-sonka\}@uiowa.edu
}

\begin{abstract}
The segmentation of soft tissues in medical images is a challenging problem due to the weak boundary, large deformation and serious mutual influence. We present a novel method incorporating both the shape and appearance information in a 3-D graph-theoretic framework to overcome those difficulties for simultaneous segmentation of prostate and bladder. An arc-weighted graph is constructed corresponding to the initial mesh. Both the boundary and region information is incorporated into the graph with learned intensity distribution, which drives the mesh to the best fit of the image. A shape prior penalty is introduced by adding weighted-arcs in the graph, which maintains the original topology of the model and constraints the flexibility of the mesh. The surface-distance constraints are enforced to avoid the leakage between prostate and bladder. The target surfaces are found by solving a maximum flow problem in low-order polynomial time. Both qualitative and quantitative results on prostate and bladder segmentation were promising, proving the power of our algorithm.
\end{abstract}

\section{Introduction}

Automated segmentation of medical image has increasingly become a valuable tool for medical diagnosis and treatment planning. Though intensive research has been done, accurate segmentation of 3-D soft tissues is still a challenging problem. The main difficulties lie in the following aspects [1/2]: First, soft tissues often present a large variation in both shape and size. Second, the target objects often lack strong boundaries and have similar intensity information. Third, many soft tissues have serious mutual influence between each other.

To overcome those difficulties, many methods have been proposed to employ both learned shape and appearance information. One of the widely used methods

\footnotetext{
* This work was supported in part by NSF grants CCF-0830402 and CCF-0844765, and NIH grants R01-EB004640 and K25-CA123112.
}

T. Jiang et al. (Eds.): MICCAI 2010, Part III, LNCS 6363, pp. 172 180, 2010.
(C) Springer-Verlag Berlin Heidelberg 2010 
is the AAM model of Cootes et al. [3. The shape and appearance of an object is modeled using principal component analysis (PCA). Freedman et al. [1] presented a segmentation algorithm based on matching probability distributions from a learned model of shape and appearance to the image. Rousson et al. developed a method to incorporate both shape and appearance model using bayesian formulation [4]. The deformable shape model with shape prior information was employed in [5] and 6] for prostate and bladder segmentation. Recently, graph-based methods with a global optimality guarantee have attracted a lot of attention. Freedman et al. 7 proposed an interactive shape prior segmentation based on graph cut algorithms. Malcolm et al. 8] employed an iterative graph cut framework with the prior shape information learned from kernel PCA. Besbes et al. 9] used an incomplete graph representation for shape modeling and aimed to optimize the connectivity of the graph nodes.

In this paper, we introduce a novel approach incorporating both shape information and object intensity distribution information for prostate and bladder segmentation. Our method is developed based on the framework reported by Kang et al. [1011] and Qi et al.[12. In [12, two coupled geometric graphs are constructed for the prostate and the bladder, respectively. The weights of both graph nodes and arcs are utilized to represent edge-based image energy and a linear soft smoothness penalty. To avoid the possible overlapping of the prostate and the bladder, the hard surface interacting constraints are enforced in the partially interacting regions. The surface segmentation problem is then transformed into that of finding a minimum-cut in a transformed graph.

While the proposed method in [12] was successfully applied for simultaneous segmentation of the prostate and the bladder, it still has several limitations. First, only gradient-based edge weight is employed in the original formulation, which often performs poorly in the presence of weak boundaries, e.g., multiple adjacent objects with similar intensity profiles. Second, only a linear soft smoothness penalty is considered, which limits the flexibility of the shape constraints. In this work, we show how to incorporate the learned intensity distribution information into the graph search framework by using both boundary energy and region energy terms. A convex shape penalty function is also enforced, which allows the incorporation of a wider spectrum of constraints.

\section{Optimal Graph Search with Appearance and Shape Information}

\subsection{Graph Modeling}

Our method is mainly based on the graph search framework proposed in [12]. For completeness, we briefly review the framework. As the first step, two initial models are constructed for the prostate and the bladder using the same method as described in [12. Based on these two initial models, two triangulated meshes $M_{1}\left(V_{1}, E_{1}\right)$ and $M_{2}\left(V_{2}, E_{2}\right)$ are constructed respectively, where $V_{i}(i \in 1,2)$ denotes the vertex set of $M_{i}$ and $E_{i}$ denotes the edge set of $M_{i}$. Fig. 11(a),(b) 


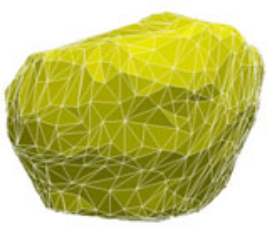

(a)

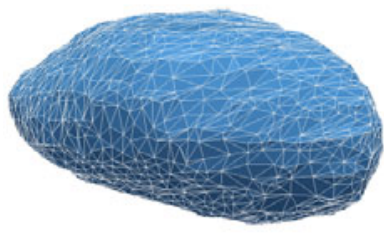

(b)

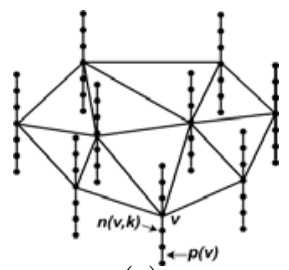

(c)

Fig. 1. (a) Triangulated mesh for the prostate. (b) Triangulated mesh for the bladder. (c) Corresponding graph construction. $p(v)$ represents the column with respect to the vetex $v$. Dots represent nodes $n \in G_{i}$.

show the constructed meshes for the prostate and the bladder. The weighted graph $G_{i}(N, A)$ is built from the mesh $M_{i}$ as follows. For each vertex $v \in V_{i}$, a column of $K$ nodes $n(v, k)$ is created in $G_{i}$, denoted by $p(v)$ (Fig. 1(c)). The positions of nodes reflect the positions of corresponding voxels in the image domain. The length of the column is set according to the required search range. The number of nodes $K$ on each column is determined by the required resolution. The direction of the column is set as the triangle normal. The nodes on the same column are connected by the directed arc from $n(v, k)$ to $n(v, k-1)$ with infinity weight. Each column also has a set of neighbors, i.e., if $\left(v_{x}, v_{y}\right) \in E_{i}$, then $p\left(v_{x}\right)$ and $p\left(v_{y}\right)$ are neighboring columns. The feasible surface $S_{i}$ in the graph $G_{i}$ is defined as the surface containing exact one node in each column. To avoid the overlapping of two target surfaces, a "partially interacting area" is defined according to the distance between two meshes, which indicates that the two target surfaces may mutually interact each other at that area. To model the interaction relation, the two graphs $G_{1}$ and $G_{2}$ "share" some common node columns in that partially interacting area, and the target surfaces $S_{1}$ and $S_{2}$ both cut those columns, as shown in Fig. $2 \mathrm{a}$. In addition, the distance between the two surfaces at the interacting area is required to be at lease $\delta_{l}$ voxels and at most $\delta_{h}$ voxels, which prevents the leakage between prostate and bladder. This surface distance constraint is incorporated by adding inter-surface arcs between corresponding nodes on those columns at the interacting region (Fig. 20 b).

The optimal set $S$ of two surfaces corresponding to the prostate and the bladder can then be found by minimizing the following energy:

$$
E(S)=\sum_{i=1}^{2} E_{\text {boundary }}\left(S_{i}\right)+\sum_{i=1}^{2} E_{\text {shape }}\left(S_{i}\right)
$$

The boundary energy term serves as an external force, which drives the mesh towards the best fit to the image data. The shape energy term functions as an internal force, which keeps the shape of the original model and restricts the flexibility of the mesh. In the original framework, a gradient-based edge cost is employed for boundary information, which may fail for surface detection when no strong boundary exists. For shape energy term, a linear soft smoothness 
shape compliance is employed. The linear function limited the flexibility of the constraints. In the subsequent sections, we show how to incorporate the learned appearance information using both the boundary energy term and an additional region energy term. Specifically, two surfaces for the prostate and the bladder naturally divide the volume into 3 regions denoted by $R_{0}, R_{1}$ and $R_{2}$, which corresponds to the region enclosed by the prostate surface $S_{1}$, one between $S_{1}$ and the bladder surface $S_{2}$ at the partially interacting area, and the region enclosed by $S_{2}$, respectively. Our region energy term $E_{\text {region }}\left(R_{i}\right)$ reflects the region property of all voxels inside $R_{i}$. In addition, we incorporate a shape prior penalty using a convex function. Thus, the energy function used in this paper is, as follows.

$$
E(S)=\sum_{i=1}^{2} E_{\text {boundary }}\left(S_{i}\right)+\sum_{i=0}^{2} E_{\text {region }}\left(R_{i}\right)+\sum_{i=1}^{2} E_{\text {shape }}\left(S_{i}\right)
$$

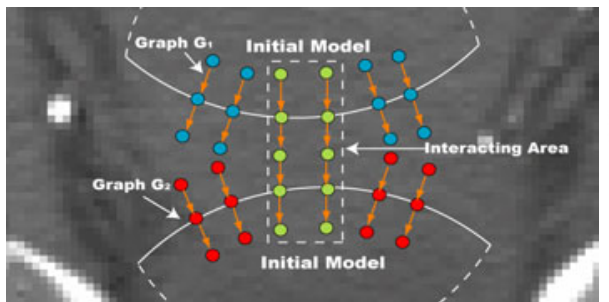

(a)

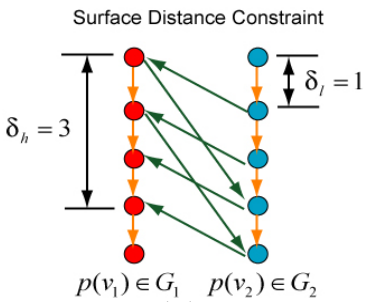

(b)

Fig. 2. (a) Graph construction for mutually interacting objects. An example 2-D slice is presented. Note that in the interacting region, for each column with green nodes, there actually exists two columns with the same position, one for Graph $G_{1}$, one for Graph $G_{2}$.(b) Inter-surface arcs are added between the column $p\left(v_{1}\right) \in G_{1}$ and the column $p\left(v_{2}\right) \in G_{2} \cdot p\left(v_{1}\right)$ and $p\left(v_{2}\right)$ have the same position in the interacting region. The surface distance constraint is set as $1 \leq S_{1}-S_{2} \leq 3$.

\subsection{Boundary Energy Design}

For each node $n(v, k) \in G_{i}$, we have an on-surface cost $c_{S_{i}}(v, k)$ with respect to surface $S_{i}$, which is inversely related to the possibility that the node belongs to the target surface $S_{i}$. The boundary energy term $E_{\text {boundary }}\left(S_{i}\right)$ is defined as the summation of the on-surface costs associated with all nodes on surface $S_{i}$, i.e., $E_{\text {boundary }}\left(S_{i}\right)=\sum_{n \in S} c_{S_{i}}(v, k)$. The on-surface weight of node $n(v, k)$, denoted by $w_{S_{i}}(v, k)$, is then assigned such that the total weight of a closed set in the graph $G_{i}$ equals to the boundary energy $E_{\text {boundary }}\left(S_{i}\right)$ of the corresponding surface $S_{i}$ :

$$
w_{S_{i}}(v, k)= \begin{cases}c_{S_{i}}(v, k) & \text { if } k=0 \\ c_{S_{i}}(v, k)-c_{S_{i}}(v, k-1) & \text { if } 0<k \leq K-1\end{cases}
$$

The on-surface cost function design plays a key role in accurate surface delineation. In soft-tissue segmentation, there is often no clear boundary information 
between adjacent tissues. The intensity gradient at the interface of adjacent tissues is also low. To overcome the difficulty, we incorporate the object class-uncertainty information into our cost function. Given a prior knowledge of intensity probability distributions of each object, the class-uncertainty can be computed for each intensity value, which yields the uncertainty level of the classification. Suppose the image is divided into $m$ objects, denoted by $o_{i}, i \in[0,1, \ldots, m-1]$. For any given node $n(v, k)$ with image intensity $g(v, k)$, the posteriori probability $p\left(o_{i} \mid g\right)$ is obtained from the training set using Bayes rules. The uncertainty measure for the classification that $n(v, k)$ with an intensity of $g$ falls into any object is the entropy of all posteriori probability values, with the form: $h(g)=\sum_{i}-p\left(o_{i} \mid g\right) \log \left(p\left(o_{i} \mid g\right)\right)$. As demonstrated in [1314, intensities with high class uncertainty measures are tend to appear close to object boundaries rather than inside homogeneous regions. Combined with the gradient information, the uncertainty measure is expected to provide an enhanced on-surface cost at the locations absent a clear boundary. Let $\nabla(v, k)$ denote the gradient magnitude at node $n(v, k)$, our boundary cost function has the following form:

$$
c_{S_{i}}(v, k)=-(\nabla(v, k)+\alpha h(g(v, k)))
$$

where $\alpha$ is a constant parameter. Here we set $\alpha=0.5$ according to the experiments on the training set.

\subsection{Incorporation of Region Information}

The graph search framework allows easy incorporation of region information by assigning proper weights for the graph nodes. As described in Section 2.1, $R_{0}$, $R_{1}$, and $R_{2}$ denote the region enclosed by the prostate surface $S_{1}$, one between $S_{1}$ and the bladder surface $S_{2}$ at the partially interacting area, and the region enclosed by $S_{2}$, respectively. For each node $n(v, k)$, the in-region cost associated with $R_{i}$ is assigned as $c_{R_{i}}(v, k)(i=0,1,2)$. The region energy term is then defined as the following form: $E_{\text {region }}\left(R_{i}\right)=\sum_{n(v, k) \in R_{i}} c_{R_{i}}(n(v, k))$. The inregion weight of node $n(v, k)$ in graph $G_{i}$, denoted by $w_{R_{i}}(v, k)$, is assigned such that the total weight of a closed set in graph $G$ equals to the region energy term $\sum_{i=0}^{2} E_{\text {region }}\left(R_{i}\right)$ (with a constant difference) [15]:

$$
w_{R_{i}}(v, k)=c_{R_{i-1}}(n(v, k))-c_{R_{i}}(n(v, k))
$$

For region cost design, the posterior probability learned from the training set is used with the form:

$$
c_{R_{i}}(n(v, k))=-p\left(n(v, k) \in R_{i} \mid g(v, k)\right), i \in\{0,1,2\}
$$

\subsection{Shape Constraints}

The shape energy keeps the topology of the original mean shape. In our framework, two types of shape constraints are enforced: the hard shape constraint and the shape-prior penalties. 
Suppose $p\left(v_{x}\right)$ and $p\left(v_{y}\right)$ are neighboring columns in the graph. If $n\left(v_{x}, k_{1}\right)$ of $p\left(v_{x}\right)$ and $n\left(v_{y}, k_{2}\right)$ of $p\left(v_{y}\right)$ are nodes on the surface $S_{i}$, we let $S_{i}\left(v_{x}\right)=k_{1}$ and $S_{i}\left(v_{y}\right)=k_{2}$. Then the hard shape constraint is defined as follows: $\Delta_{l} \leq$ $S_{i}\left(v_{x}\right)-S_{i}\left(v_{y}\right) \leq \Delta_{h} . \Delta_{l}$ and $\Delta_{h}$ are specified shape constraint parameters between $p\left(v_{x}\right)$ and $p\left(v_{y}\right)$. To enforce the hard shape constraint, a directed arc with $+\infty$ weight is put from each node $n\left(v_{x}, k\right)$ to the node $n\left(v_{y}, k-\Delta_{h}\right)$. On the other hand, we have a directed arc with $+\infty$ from the node $n\left(v_{y}, k\right)$ to $n\left(v_{x}, k+\Delta_{l}\right)$.

The shape-prior penalties are set as $f\left(S_{i}\left(v_{x}\right)-S_{i}\left(v_{y}\right)\right)$, where $f(\cdot)$ is a convex function penalizing the shape changes of $S_{i}$ between neighboring column $p\left(v_{x}\right)$ and $p\left(v_{y}\right)$. Based on this definition, our shape energy term has the form:

$$
E_{\text {shape }}\left(S_{i}\right)=\sum_{\left(v_{x}, v_{y}\right) \in E_{i}} f\left(S_{i}\left(v_{x}\right)-S_{i}\left(v_{y}\right)\right)
$$

To incorporate the shape energy, additional inter-column arcs are employed. The main idea is to distribute the convex shape prior penalty $f\left(S_{i}\left(v_{x}\right)-S_{i}\left(v_{y}\right)\right)$ to the corresponding cuts between the columns in $G$ based on the (discrete equivalent of ) second derivative of $f(\cdot), f^{\prime \prime}(h)=[f(h+1)-f(h)]-[f(h)-$ $f(h-1)$ ], as described in [1617]. Since $f(h)$ is a convex function, $f^{\prime \prime}(h) \geq 0$. For each $h=\Delta_{l}+1, \Delta_{l}+2, \ldots, \Delta_{h}-1$, if $f^{\prime}(h) \geq 0$, an arc from $n\left(v_{x}, k\right)$ to $n\left(v_{y}, k-h\right)$ is assigned with an arc-weight of $f^{\prime \prime}(h)$. If $f^{\prime}(h) \leq 0$, an arc from $n\left(v_{y}, k\right)$ to $n\left(v_{x}, k+h\right)$ is assigned with the weight of $f^{\prime \prime}(h)$ (Fig. 3 (b)). Using this construction, the total weight of the arcs spanning between two neighboring columns $p\left(v_{x}\right)$ and $p\left(v_{y}\right)$ equals to the shape prior penalty $f\left(S_{i}\left(v_{x}\right)-S_{i}\left(v_{y}\right)\right)$.

For prostate and bladder segmentation, a second order shape prior penalty is employed with the form: $f(h)=\beta \cdot h^{2}$, where $\beta$ is a constant parameter learned from the training set. In this project $\beta=5$.

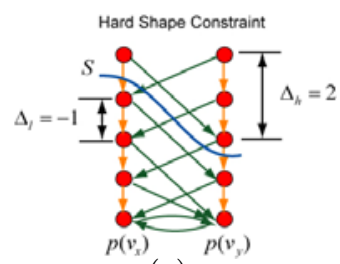

(a)

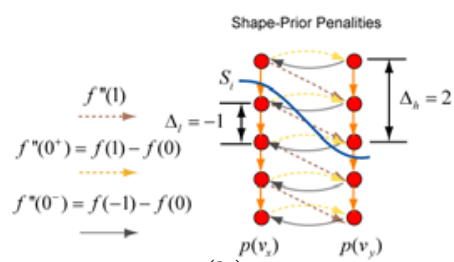

(b)

Fig. 3. Arc construction for shape constraints. (a) Hard shape constraints. (b) Shapeprior penalties. Note that here we suppose $f^{\prime}(0)=0 . S$ cuts the arcs with a total weight of $f(2)$.

\subsection{Optimization}

Once the graph is constructed, an optimal cut $\mathcal{C}^{*}=\left(B^{*}, \bar{B}^{*}\right)\left(B^{*} \cup \bar{B}^{*}=N\right)$ can be found in $G(N, A)$, minimizing the total weight of nodes in $B^{*}$ and the total arc weight of $\mathcal{C}^{*}$. The optimal cut actually corresponds to the optimal set $S$ of 
two surfaces $S_{1}$ and $S_{2}$, which minimizes the total energy $E(S)$. As described in [18, the optimal cut can be found by solving a maximum flow problem in a transformed graph $G^{\prime}$ in a low-order polynomial time.

\section{Experiments and Results}

The experiments were conducted for simultaneous segmentation of prostate and bladder. 3-D CT images from different patients with the prostate cancer are used. The image size ranges from $80 \times 120 \times 30$ to $190 \times 180 \times 80$ voxles. The image spacing resolution ranged from $0.98 \times 0.98 \times 3.00 \mathrm{~mm}^{3}$ to $1.60 \times 1.60 \times$ $3.00 \mathrm{~mm}^{3}$. Out of 21 volumes, 8 were randomly selected as the training data and our segmentation was performed on the remaining 13 datasets. Our workflow mainly consisted of three major steps. Step 1. Initial model construction. We use the similar method as described in 12 . Since the prostate often shows a good statistical coherency in shape among different datasets [4, a mean shape is obtained from the training set and roughly fitted into the image as the initial model. For bladder, a 3-D geodesic method is employed, the result provides the basic topological structure information. Step 2. Iterative single mesh deformation for prostate and bladder, separately. Using iterative graph search, we can find target surfaces which are far away from the initial model. In our experiment, we set iteration times as 3, which is proved enough according to the experimental result. Step 3. Simultaneous graph search for bladder and prostate. The possible overlapping was avoided by adding surface distance constraint between the two meshes.

For quantitative validation, the result was compared with the expert-defined manual contours. For volumetric error measurement, the Dice similarity coefficient (DSC) was computed using $D=2\left|V_{m} \bigcap V_{c}\right| /\left(\left|V_{m}\right|+\left|V_{c}\right|\right)$, where $V_{m}$ denotes the manual volumetric result and $V_{c}$ denotes the computed result. For surface distance error, both mean and the maximum unsigned surface distance error were computed for the bladder and the prostate surfaces between the computed result and the manual delineation. The result is shown in Table 1. Compared with the result reported in [12, the unsigned surface distance error has improved from $1.38 \pm 1.08 \mathrm{~mm}$ to $1.01 \pm 0.94 \mathrm{~mm}$ for the prostate and from $1.04 \pm 1.00 \mathrm{~mm}$ to $0.99 \pm 0.77 \mathrm{~mm}$ for the bladder.

Table 1. Overall quantitative results. Mean \pm SD in $m m$ for the unsigned surface distance error.

\begin{tabular}{llll}
\hline Surface & $D S C$ & Mean $(\mathrm{mm})$ & $\operatorname{Maximum}(\mathrm{mm})$ \\
\hline Prostate & 0.797 & $1.01 \pm 0.94$ & $5.46 \pm 0.96$ \\
Bladder & 0.900 & $0.99 \pm 0.77$ & $5.88 \pm 1.29$ \\
\hline
\end{tabular}

For a visual performance assessment, the illustrative result with both computed contours and manual contours was displayed in Fig 4(a). The 3-D representation was shown in Fig 4(b). 


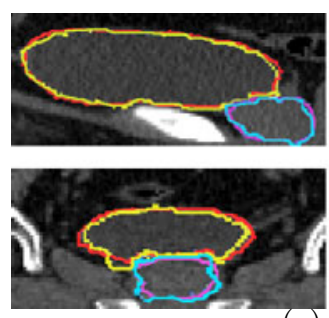

(a)
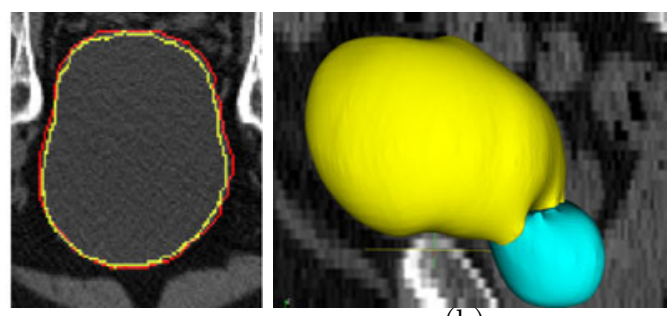

(b)

Fig. 4. (a) Sagittal, coronal and transverse slices with the computed result (blue for prostate and yellow for bladder) and the manual result (magenta for prostate and red for bladder). (b) 3-D representation of the prostate (blue) and the bladder (yellow).

The execution time on a WinXP PC $(2.13 \times 2 \mathrm{GHz}, 2 \mathrm{~GB}$ memory $)$ was approximately 8 minutes per dataset.

\section{Discussion and Conclusion}

We have presented how to incorporate boundary, region and shape information into an optimal 3-D graph search framework for multiple objects segmentation. The general formulation has been adapted to the simultaneous segmentation of the prostate and the bladder. Both qualitative and quantitative validation were employed. The result shows the power of our algorithm.

\section{References}

1. Freedman, D., Radke, R.J., Zhang, T., Jeong, Y., Lovelock, D.M., Chen, G.T.Y.: Model-based segmentation of medical imagery by matching distributions. IEEE Trans. Medical Imaging 24(3), 281-292 (2005)

2. Vu, N., Manjunath, B.S.: Shape prior segmentation of multiple objects with graph cuts. In: Proc. IEEE International Conference on Computer Vision and Pattern Recognition, pp. 1-8 (2008)

3. Cootes, T.F., Edwards, G.J., Taylor, C.J.: Active appearance models. IEEE Trans. Pattern Anal. Machine Intell 23(6), 681-685 (2001)

4. Rousson, M., Khamene, A., Diallo, M., Celi, J.C., Sauer, F.: Constrained surface evolutions for prostate and bladder segmentation in CT images. In: Proc. of the First International Workshop on Computer Vision for Biomedical Image Applications, pp. 251-260 (2005)

5. Costa, M.J., Delingette, H., Novellas, S., Ayache, N.: Automatic segmentation of bladder and prostate using coupled 3D deformable models. In: Ayache, N., Ourselin, S., Maeder, A. (eds.) MICCAI 2007, Part I. LNCS, vol. 4791, pp. 252-260. Springer, Heidelberg (2007)

6. Heimann, T., Munzing, S., Meinzer, H.P., Wolf, I.: A shape-guided deformable model with evolutionary algorithm initialization for $3 \mathrm{~d}$ soft tissue segmentation. In: Karssemeijer, N., Lelieveldt, B. (eds.) IPMI 2007. LNCS, vol. 4584, pp. 1-12. Springer, Heidelberg (2007) 
7. Freedman, D., Zhang, T.: Interactive graph cut based segmentation with shape priors. In: Proc. IEEE International Conference on Computer Vision and Pattern Recognition, vol. 1, pp. 755-762 (2005)

8. Malcolm, J., Rathi, Y., Tannenbaum, A.: Graph cut segmentation with nonlinear shape priors. In: Proc. International Conference on Image Processing (2007)

9. Besbes, A., Komodakis, N., Langs, G., Paragios, N.: Shape priors and discrete mrfs for knowledge-based segmentation. In: Proc. IEEE International Conference on Computer Vision and Pattern Recognition, pp. 1295-1302 (2009)

10. Li, K., Wu, X., Chen, D.Z., Sonka, M.: Optimal surface segmentation in volumetric images - a graph-theoretic approach. IEEE Trans. Pattern Anal. Machine Intell. 28(1), 119-134 (2006)

11. Li, K., Jolly, M.P.: Simultaneous detection of multiple elastic surfaces with application to tumor segmentation in CT images. In: Proc. SPIE Medical Imaging: Image Processing, vol. 6914 (2008)

12. Song, Q., Wu, X., Liu, Y., Smith, M., Buatti, J.M., Sonka, M.: Optimal graph search segmentation using arc-weighted graph for simultaneous surface detection of bladder and prostate. In: Yang, G.-Z., Hawkes, D., Rueckert, D., Noble, A., Taylor, C. (eds.) MICCAI 2009. LNCS, vol. 5762, pp. 827-835. Springer, Heidelberg (2009)

13. Saha, P.K., Udupa, J.K.: Optimum threshold selection using class uncertainty and region homogeneity. IEEE Trans. Pattern Anal. Machine Intell. 23, 689-706 (2001)

14. Saha, P.K., Das, B., Wehrli, F.W.: An object class-uncertainty induced adaptive force and its application to a new hybrid snake. Pattern Recognition 40, 2656-2671 (2007)

15. Haeker, M., Abramoff, M.D., Wu, X., Russell, S.R., Burns, T.L., Sonka, M.: Automated 3-d intraretinal layer segmentation of macular spectral-domain optical coherence tomography images. IEEE Trans. Medical Imaging 28(9), 1436-1447 (2009)

16. Ishikawa, H.: Exact optimization for markov random fields with convex priors. IEEE Trans. Pattern Anal. Machine Intell. 25(10), 1333-1336 (2003)

17. Song, Q., Wu, X., Liu, Y., Haeker, M., Sonka, M.: Simultaneous searching of globally optimal interacting surfaces with convex shape priors. In: Proc. IEEE International Conference on Computer Vision and Pattern Recognition (2010)

18. Wu, X., Chen, D.Z.: Optimal net surface problems with applications. In: Widmayer, P., Triguero, F., Morales, R., Hennessy, M., Eidenbenz, S., Conejo, R. (eds.) ICALP 2002. LNCS, vol. 2380, pp. 1029-1042. Springer, Heidelberg (2002) 